\title{
J A \\ Opening ZIF-8: A Catalytically Active Zeolitic Imidazolate Framework of Sodalite Topology with Unsubstituted Linkers
}

Olga Karagiaridi, ${ }^{\dagger}$ Marianne B. Lalonde, ${ }^{\dagger}$ Wojciech Bury, ${ }^{\dagger, \dagger}$ Amy A. Sarjeant, ${ }^{\dagger}$ Omar K. Farha, ${ }^{*}{ }^{\dagger}$ and Joseph T. Hupp* ${ }^{* \dagger}$

${ }^{\dagger}$ Department of Chemistry and International Institute for Nanotechnology, Northwestern University, 2145 Sheridan Road, Evanston, Illinois 60208, United States

${ }^{\ddagger}$ Department of Chemistry, Warsaw University of Technology, Noakowskiego 3, 00-664 Warsaw, Poland

\section{Supporting Information}

ABSTRACT: A zeolitic imidazolate framework material of SOD topology possessing primarily unsubstituted imidazolate (im) linkers has been synthesized via solvent-assisted linker exchange (SALE) of ZIF-8. The structure of the new material, SALEM-2, has been confirmed through ${ }^{1} \mathrm{H}$ NMR and powder and single-crystal X-ray diffraction. SALEM-2 is the first example of a porous $\mathrm{Zn}(\mathrm{im})_{2}$ ZIF possessing a truly zeolitic topology that can be obtained in bulk quantities. Upon treatment with $n$-butyllithium, the open analogue exhibits Brønsted base catalysis that cannot be accomplished by the parent material ZIF-8. Additionally, it displays a different size cutoff for uptake and release of molecular guests than does ZIF-8.

\section{INTRODUCTION}

Zeolitic imidazolate frameworks $(\mathrm{ZIFs})^{1-3}$ are a subclass of metal-organic framework (MOF) ${ }^{4-11}$ materials possessing attractive structural properties and great potential for applications in chemical storage, ${ }^{12}$ chemical encapsulation and controlled delivery, ${ }^{13}$ chemical sensing, ${ }^{14}$ and chemical separations, ${ }^{15,16}$ including membrane-based separations. ${ }^{17-19}$ Structurally they are isomorphous with zeolites, a consequence of the bridging metal-imidazolate-metal angle of $\sim 145^{\circ}$ (equivalent to the average $\mathrm{Si}-\mathrm{O}-\mathrm{Si}$ angle in zeolites) ${ }^{20}$ and the ability of the substances to crystallize in mostly uninodal topologies characteristic of zeotype materials. ZIFs possess several attractive features relative to zeolites, such as higher surface area due to their intrinsically lower density, and increased structural tailorability due to the presence of organic linkers. However, the potential of ZIFs remains to be realized. Just over 100 distinct ZIF structures had been reported by 2010, as compared to over 190 known zeolites. ${ }^{2}$ Moreover, many of these structures have only been obtained at the scale of a few crystals; as a consequence, experimental data concerning bulk properties and performance in potential industrial applications have been collected for only a very small subset of all known ZIFs, and an infinitesimal fraction of potential ZIF compounds.

In a manner reminiscent of that used in preparing zeolites, ${ }^{21}$ the distinct topologies available for ZIFs can be obtained through a judicious choice of structure-directing agents (SDAs). The role of the SDAs is often fulfilled by the substituents on the imidazolate linkers, as the interactions between them have been shown to foster the formation of topologies such as SOD, RHO, ANA, and LTA. ${ }^{22-24}$ Notably,

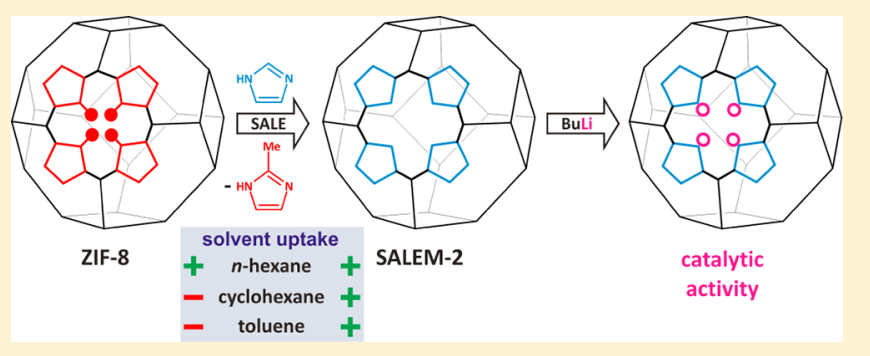

atilization of bulky substituents leads to the assembly of large cages and structures that lack precedent among purely inorganic zeolites. ${ }^{25}$ While sometimes enabling the synthesis of ZIFs with topologies leading to otherwise unstable or metastable open structures, the substituents may also block several or all of the apertures and thus detract from porosity. To give a few examples, the 2-ethyl substituent that gives ZIF14 its ANA topology also renders ZIF-14 essentially nonporous, ${ }^{22}$ while the purinate linkers in ZIF-20 (LTA) restrict the aperture of ZIF-20 to just $2.8 \AA$, making its pores inaccessible even to molecular nitrogen. ${ }^{24}$

Unsubstituted imidazolate ( $\mathrm{im}$ ) is an attractive ZIF linker, as its incorporation into an open architecture should lead to a material with large voids, unobstructed apertures, and appreciable surface area. Not surprisingly, substantial work has been done on synthesizing zinc-based ZIFs with im linkers. The first crystalline polymeric structure with the formula $\mathrm{Zn}(\mathrm{im})_{2}$ was synthesized in 1980 and formed as the dense zni net. ${ }^{26}$ Twenty years later, Tian and co-workers obtained seven different $\mathrm{Zn}(\mathrm{im})_{2}$ polymorphs by using solvents capable of acting as templates. ${ }^{27}$ They were quick to notice that dense, nonporous nets such as nog, BCT, and cag are thermodynamically stable and easy to isolate, whereas more porous frameworks such as DFT and GIS can only be obtained on a small scale through liquid diffusion and often are minor kinetic products. The subsequent work of Yaghi and co-workers in creating $\mathrm{Zn}(\mathrm{im})_{2}$ isomers complements those findings; bulk quantities of ZIF-1 (BCT) and ZIF-4 (cag) could be

Received: September 4, 2012

Published: October 22, 2012 
Table 1. Structural Characteristics of All $\mathrm{Zn}(\mathrm{im})_{2}$ Polymorphs Reported Thus Far ${ }^{a}$

\begin{tabular}{|c|c|c|c|c|c|c|}
\hline $\mathrm{Zn}(\mathrm{im})_{2}$ name or CCDC & ref & topology & $\begin{array}{l}\text { largest aperture diameter } \\
{[\AA \AA]^{2}}\end{array}$ & $\begin{array}{l}\text { density } \\
{\left[\mathrm{g} / \mathrm{cm}^{3}\right]}\end{array}$ & $\begin{array}{l}\text { total energy relative to the zni topology } \\
\qquad[\mathrm{kJ} / \mathrm{mol}]^{29}\end{array}$ & comments \\
\hline IMIDZB01 & 26 & zni & 3.6 & 1.56 & 0 & \\
\hline ZIF-4 & 23 & cag & 2.1 & $1.44^{b}$ & 6.33 & nonporous \\
\hline VEJYUF01 & 27 & cag & 0.8 & $1.43^{b}$ & & $\begin{array}{l}\text { collapses after guest } \\
\text { removal }\end{array}$ \\
\hline ZIF-1 & 23 & BCT & 6.3 & $1.47^{b}$ & 7.92 & \\
\hline VEJYEP01 & 27 & BCT & 2.2 & $1.47^{b}$ & & $\begin{array}{l}\text { collapses after guest } \\
\text { removal }\end{array}$ \\
\hline ZIF-10 & 23 & MER & 8.2 & $0.79^{b}$ & 11.1 & few crystals scale \\
\hline $\begin{array}{l}\text { hypothetical SOD } \\
\mathrm{Zn}(\mathrm{im})_{2}\end{array}$ & & SOD & & 0.83 & 11.8 & $\mathrm{n} / \mathrm{a}$ \\
\hline ZIF-3 & 23 & DFT & 4.6 & $1.09^{b}$ & 12.2 & few crystals scale \\
\hline HIFVOI & 27 & DFT & 6.6 & $0.93^{b}$ & & $\begin{array}{l}\text { collapses after guest } \\
\text { removal }\end{array}$ \\
\hline ZIF-6 & 23 & GIS & 1.5 & $0.79^{b}$ & 12.6 & few crystals scale \\
\hline HIFVUO & 27 & GIS & 5.2 & 0.82 & & few crystals scale \\
\hline ZIF-2 & 23 & BCT & 6.4 & $1.39^{b}$ & 13.4 & few crystals scale \\
\hline VEJYIT01 & 27 & BCT & 5.4 & $1.43^{b}$ & & few crystals scale \\
\hline ZIF-64 & 12 & BCT & 2.5 & $1.45^{b}$ & & few crystals scale \\
\hline EQOCOC & 30 & coi & 2.5 & $1.51^{b}$ & $\mathrm{n} / \mathrm{a}$ & \\
\hline HICGEG & 27 & zec & 5.0 & $1.08^{b}$ & $\mathrm{n} / \mathrm{a}$ & $\begin{array}{l}\text { collapses after guest } \\
\text { removal }\end{array}$ \\
\hline HIFWAV & 27 & nog & 4.7 & $1.26^{b}$ & $\mathrm{n} / \mathrm{a}$ & \\
\hline KUMXEW & 28 & $\mathrm{n} / \mathrm{a}$ & $\mathrm{n} / \mathrm{a}$ & 1.60 & $\mathrm{n} / \mathrm{a}$ & nonporous \\
\hline
\end{tabular}

${ }^{a}$ For the ZIFs that do not have a conventional name, the CCDC code has been provided. ${ }^{b}$ Density includes residual solvent guest molecules within the crystal.

successfully obtained, whereas less dense ZIF-3 (DFT), ZIF-6 (GIS), and ZIF-10 (MER) were only synthesized on a scale sufficient to collect single-crystal data. ${ }^{23}$ In summary, all of the $\mathrm{Zn}(\mathrm{im})_{2}$ polymorphs synthesized to date, a remarkable 17 total, are either too dense to be useful as molecular sorbents, collapse upon removal of guest solvent molecules, and/or have only been obtainable on the scale of a few crystals (Table 1). ${ }^{12,20,23,26-28}$

Despite substantial effort, notably missing from known $\mathrm{Zn}(\mathrm{im})_{2}$ polymorphs is the unsubstituted imidazolate version of ZIF-8, ${ }^{23}$ also sometimes called MAF-4. ${ }^{22}$ A high-void-volume compound, ZIF-8 possesses large spherical cavities but small apertures (ca. $3.4 \AA$ diameter, if expansion via linker torsional motion $^{31}$ is ignored). This iconic material features sodalite (SOD) topology and consists of $\mathrm{Zn}^{2+}$ and 2-methyl-imidazolate ( mim) in 1:2 stoichiometry. Remarkably easy to assemble in particulate, ${ }^{18,23}$ nanoparticulate, ${ }^{32,33}$ thin-film, ${ }^{14,34,35}$ 2-D opal, $^{36}$ and even membrane form, ${ }^{37}$ ZIF-8 is the single most extensively studied ZIF material, both from a fundamental ${ }^{38,39}$ and from an applied, functional perspective. ${ }^{14,16,40-42}$ Table 1 (energy data from Cheetham and co-workers) ${ }^{29}$ shows why the SOD form (ZIF-8-like form) of $\mathrm{Zn}(\mathrm{im})_{2}$ has proven so elusive: it is less stable thermodynamically than any of the $\mathrm{Zn}(\mathrm{im})_{2}$ polymorphs that have been previously synthesized in macroscopic quantities. Furthermore, it is also less stable than some of the comparatively high-energy polymorphs that have been obtained only in trace quantities. Additionally, its density is substantially less than that of any of the previously described, scalable polymorphs, as well as most of the nonscalable ones. Thus, a direct solvothermal, ionothermal, ${ }^{43}$ or similar synthesis strategy for the SOD form of $\mathrm{Zn}(\mathrm{im})_{2}$ would appear unlikely to succeed.

Recently, Choe and co-workers described an indirect route to desired pillared-paddlewheel MOFs. ${ }^{44}$ Briefly, a known pillaredpaddlewheel compound was exposed to a solution containing a desired alternative and shorter candidate pillaring linker molecule. Under appropriate conditions (solvent, temperature, time, and concentration of replacement linker), they were able to observe complete pillar exchange. Lee et al. showed that pillared-paddlewheel (3D) to layered (2D) MOF conversion could be achieved via exchange of a ditopic linker (bodipy) with a monotopic molecule (pyridine).$^{45}$ Recently, we showed that a similar solvent-assisted linker exchange (SALE) approach could be used to transform CdIF-4 (a ZIF featuring large apertures and 1D channels defined by a RHO topology) into isostructural analogues. ${ }^{46}$ We reasoned that extension of SALE to ZIF-8 might provide us with an im-containing analogue. ZIF-8 features smaller apertures and stronger coordination bonds than CdIF-4, and chemically is extraordinarily robust, ${ }^{23,47}$ and so might be expected to resist SALE. However, recent work by Cohen and co-workers, ${ }^{48}$ demonstrating linker exchange within the exceptionally stable MOF, UiO-66, ${ }^{49}$ suggested to us that SALE was worth attempting.

Here, we report the synthesis of a previously unattainable (approximate) $\mathrm{Zn}(\mathrm{im})_{2}$ polymorph, SALEM-2, possessing SOD topology via solvent-assisted majority exchange (up to $85 \%$ ) of $\mathrm{mim}$ for $\mathrm{im}$ within ZIF-8 (Scheme 1). SALEM-2 retains the porosity of the parent compound ZIF-8, but offers a larger size-cutoff for uptake of molecular guests. Additionally, SALE opens up a secondary aperture that is unusably small in the parent material. The imidazolate linker renders SALEM-2 capable of performing Brønsted base catalysis upon treatment with $n$-butyllithium. The parent ZIF-8 is catalytically inactive toward this reaction. Thus, we show below that SALE can be used to create in useful amounts new high-energy ZIFs capable of performing new functions, even when the parent materials are as chemically robust, and heretofore seemingly inert, as ZIF-8. 
Scheme 1. (a) 3D Representation of the Tiling of the SOD Topology; (b) View along the Crystallographic a Axis; and (c) View along [111] Direction of ZIF-8 (Left) and SALEM2 (Right)

a)

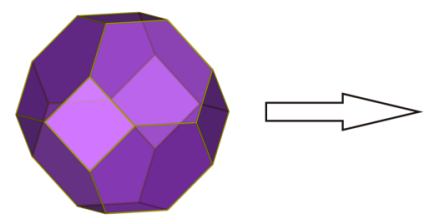

III

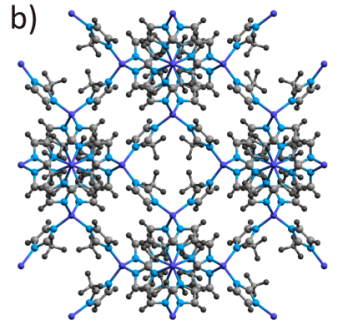

c) III

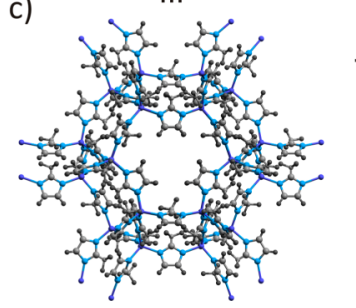

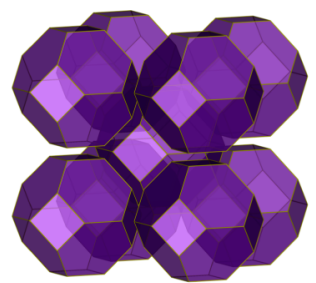

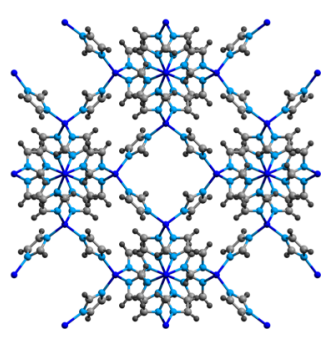

III

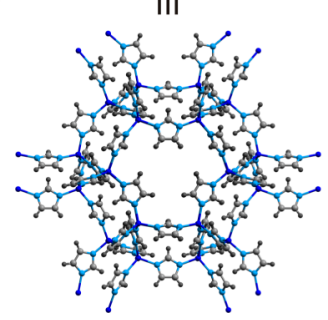

\section{EXPERIMENTAL SECTION}

Materials. Zinc nitrate hexahydrate (Fluka, 99\%), n-butanol (Aldrich, $\geq 99.4 \%$ ), $N, N^{\prime}$-dimethylformamide (Macron, 99.8\%), nitric acid (Aldrich, 70\%), ethanol (Macron, 96\%), imidazole (Him) (Aldrich, 99\%), 2-methylimidazole (Hmim) (Aldrich, 99\%), E-hex-4ene-3-one (Aldrich, $\geq 90 \%$ ), benzyl alcohol (Aldrich, $\geq 98 \%$ ), $n$-hexane (Aldrich >95\%), cyclohexane (Aldrich, 99.9\%), toluene (Aldrich, 99.8\%), and deuterated sulfuric acid (Cambridge, 96-98\% solution in $\mathrm{D}_{2} \mathrm{O}$ ) were used as received. Tetrahydrofuran, toluene, and methanol used for catalysis experiments were purified by passage through a bed of activated alumina. ${ }^{50}$

Synthesis of ZIF-8. $350 \mathrm{mg}$ of $\mathrm{Zn}\left(\mathrm{NO}_{3}\right)_{2} \cdot 6 \mathrm{H}_{2} \mathrm{O}$ and $200 \mathrm{mg}$ of 2methylimidazole were placed in a $20 \mathrm{~mL}$ screw-top vial and dissolved in $15 \mathrm{~mL}$ of DMF. Three drops of $\mathrm{HNO}_{3}$ were added to a mixture with a Pasteur pipet, and complete dissolution was achieved by sonication. The vial was capped and placed in an oven at $120^{\circ} \mathrm{C}$ for 24 h. ZIF-8 crystals were collected and washed with DMF. The crystals were stored in DMF until needed for experiments.

Synthesis of SALEM-2 via SALE. Imidazole ( $200 \mathrm{mg}, 2.94 \mathrm{mmol})$ was placed in a $20 \mathrm{~mL}$ microwave vial and dissolved in $n$-butanol $(20$ $\mathrm{mL})$ by sonication. $100 \mathrm{mg}$ of activated ZIF-8 crystals $(0.44 \mathrm{mmol})$ was immersed in the resulting solution. The vial was capped and placed in an isothermal oven at $100{ }^{\circ} \mathrm{C}$. The progress of the ligand exchange reaction was monitored by ${ }^{1} \mathrm{H} \mathrm{NMR}$, and the reaction was stopped once $\sim 85 \%$ ligand exchange was achieved ( 7 days after initiation). For the reverse SALE experiment, $200 \mathrm{mg}$ of 2methylimidazole was placed in a microwave vial and dissolved in 10 $\mathrm{mL}$ of $n$-butanol solution. $100 \mathrm{mg}$ of SALEM-2 was immersed in the resulting solution and heated in an isothermal oven at $100{ }^{\circ} \mathrm{C}$ for 6 days.

Instrumentation. ${ }^{1} \mathrm{H}$ NMR spectra were collected on a Bruker Avance III $500 \mathrm{MHz}$ spectrometer. Powder X-ray diffraction (PXRD) patterns during the synthesis of SALEM-2 were recorded on a Rigaku ATXG diffractometer equipped with an $18 \mathrm{~kW} \mathrm{Cu}$ rotating anode, MLO monochromator, and a high-count-rate scintillation detector

(measurements made over a range of $5^{\circ}<2 \theta<20^{\circ}$ in $0.05^{\circ}$ step width with a $2 \mathrm{deg} / \mathrm{min}$ scanning speed). PXRD patterns for nylon-loop mounted samples measured before and after catalysis of SALEM-2 were obtained at room temperature with a Bruker MX I $\mu \mathrm{S}$ microsource ( $\mathrm{Cu} \mathrm{K} \alpha$ radiation) and Apex II CCD detector. Samples were mounted in paratone oil. The PXRD data were collected with an area detector as rotation frames over $180^{\circ}$ in $\varphi$ at $2 \theta$ values of $12^{\circ}$, $24^{\circ}, 36^{\circ}, 48^{\circ}$, and $60^{\circ}$ and exposed for $10 \mathrm{~min}$ for each frame. At a distance of $150 \mathrm{~mm}$, the detector area covers $24^{\circ}$ in $2 \theta$. Overlapping sections of data were matched, and the resulting pattern was integrated using the Bruker APEX2 phase ID program. Powder-pattern data were treated for amorphous background scatter. Activation of ZIF-8 and SALEM-2 prior to Brunauer-Emmett-Teller (BET) measurements was done on a Quantachrome MasterPrep. Nitrogen isotherms were measured on a Micromeritics TriStar II 3020 at $77 \mathrm{~K}$. Images of the single crystals were collected using a Nikon SMZ1500 stereozoom microscope equipped with a digital camera and video monitor for visualization of crystalline samples. The single-crystal data were collected on a Bruker APEX2 V2.1-4 CCDC diffractometer with $\mathrm{Cu}$ $\mathrm{K} \alpha$ radiation $(\lambda=1.54184 \AA)$ at $100 \mathrm{~K}$. Thermogravimetric analysismass spectrometry (TGA-MS) was performed on a Mettler Toledo TGA/DSC 1 interfaced with a PC and equipped with an SEM detector using Star and Quadera software. Samples of $\sim 10 \mathrm{mg}$ were heated from 25 to $300{ }^{\circ} \mathrm{C}$ (to $800{ }^{\circ} \mathrm{C}$ for the determination of thermal stability) at a rate of $5{ }^{\circ} \mathrm{C} / \mathrm{min}$ under a nitrogen atmosphere.

NMR of SALEM-2. Approximately $5 \mathrm{mg}$ of SALEM-2 was placed in a 1.5 dram vial and dissolved in $1 \mathrm{~mL}$ of $\mathrm{D}_{2} \mathrm{SO}_{4}$ by sonication. Once a homogeneous solution was obtained, the sample was transferred to an NMR tube. ${ }^{1} \mathrm{H}$ NMR spectra were obtained by locking the sample directly to $\mathrm{D}_{2} \mathrm{SO}_{4}$. Alternatively, a 9:1 $\mathrm{D}_{2} \mathrm{O} / \mathrm{D}_{2} \mathrm{SO}_{4}(\mathrm{v} / \mathrm{v})$ solution was used as an NMR solvent (the sample was locked to $\mathrm{D}_{2} \mathrm{O}$ ).

Preparation of SALEM-2 and ZIF-8 for BET Measurements. ZIF-8 was soaked in $n$-butanol for $24 \mathrm{~h}$ and was subsequently activated on a Quantachrome MasterPrep instrument at $120{ }^{\circ} \mathrm{C}$ for $24 \mathrm{~h}$. SALEM-2 was subjected to Soxhlet extraction in ethanol for $24 \mathrm{~h}$ and subsequently activated on a Quantachrome MasterPrep instrument at $100{ }^{\circ} \mathrm{C}$ for $24 \mathrm{~h}$. Approximately $50-70 \mathrm{mg}$ was used for BET measurements.

Catalysis. This procedure was modified from Phillips et al. ${ }^{51}$ For details about catalysis using IMes and TIF-1 and monitoring by gas chromatography, see Lalonde et al. ${ }^{52}$ SALEM-2 $(2.5 \mathrm{~mol} \%)^{53}$ was added to a 2 dram oven-dried vial. The vial was sealed with a screw cap equipped with a Teflon septum, and purged with $\mathrm{N}_{2}$. Under $\mathrm{N}_{2}$, THF $(0.80 \mathrm{~mL})$ was added. The reaction was cooled to $-78{ }^{\circ} \mathrm{C}$ in a $\mathrm{CO}_{2} /$ acetone bath, and $n$-BuLi ( $16 \mu \mathrm{L}, 0.02 \mathrm{mmol}, 2.50 \mathrm{M}$ in hexanes) was added through a syringe. The reaction was allowed to warm to $20{ }^{\circ} \mathrm{C}$ by removing the vial from the dry ice/acetone bath. After $10 \mathrm{~min}$, the solvent was removed under vacuum, and the vial was backfilled with $\mathrm{N}_{2}$. A premade mixture of (E)-hex-4-ene-3-one $(92 \mu \mathrm{L}, 0.8 \mathrm{mmol})$, alcohol (97 $\mu \mathrm{L}, 2.4 \mathrm{mmol}$ for methanol, $248 \mu \mathrm{L}, 2.4 \mathrm{mmol}$ for benzyl alcohol), and toluene $(0.80 \mathrm{~mL})$ was added to the vial via syringe. Percent conversion was calculated by proton NMR $\left(\mathrm{CDCl}_{3}\right)$ using the methyl ether peak from the product (singlet, $3.33 \mathrm{ppm}, 3 \mathrm{H}$ ) for methanol, or the benzyl ether peak from the product (singlet, 4.63 $\mathrm{ppm}, 2 \mathrm{H}$ ) for the benzyl alcohol, and comparing it to the $\mathrm{C} 4$ alkene proton of the starting material (doublet, $6.08 \mathrm{ppm}, 1 \mathrm{H}$ ).

To test the heterogeneity of the reaction, the catalysis with methanol was repeated in duplicate. A small aliquot $(\sim 10 \mu \mathrm{L})$ was pulled from the surface of each reaction with a syringe after $1 \mathrm{~h}$, and percent conversion was measured by proton NMR $\left(\mathrm{CDCl}_{3}\right)$ at approximately $12 \%$ conversion for each duplicated reaction according to the method above. One reaction was brought into a glovebox under argon atmosphere and passed through a Millipore PVDF $0.1 \mu \mathrm{m}$ syringe filter, and the other reaction was allowed to continue to completion with contact to the catalyst. After $24 \mathrm{~h}$, percent conversion was calculated for each reaction. The reaction that had been syringefiltered (removed from the catalyst) remained at $12 \%$ conversion, whereas the control reaction continued to $55 \%$ conversion to product. 


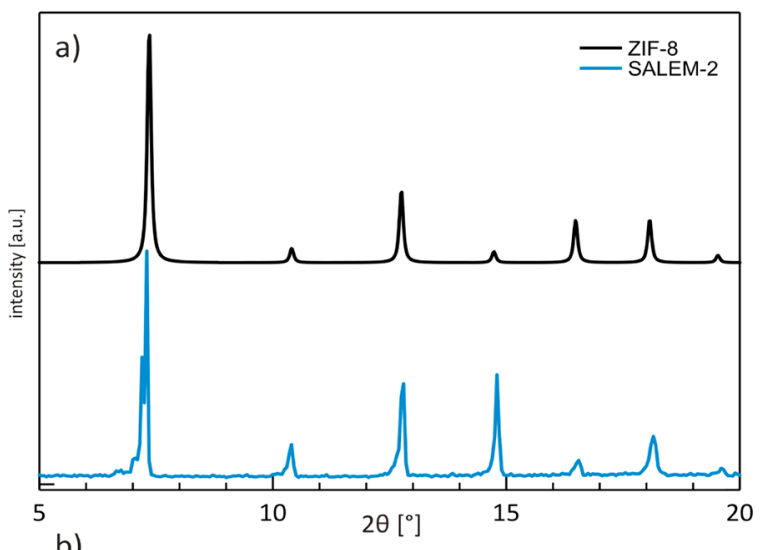

b)

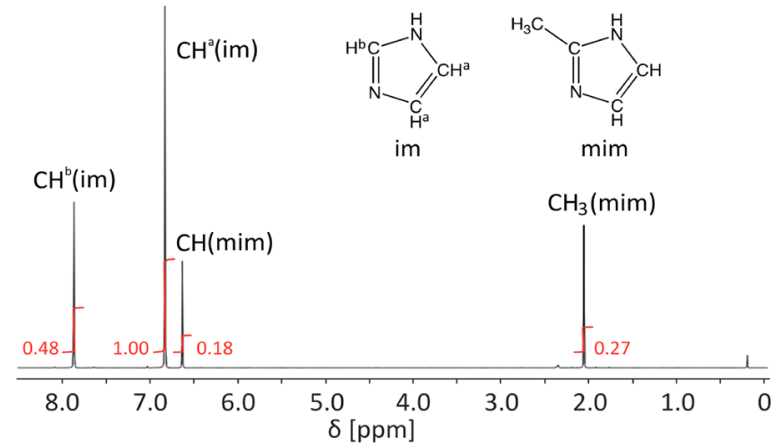

c)

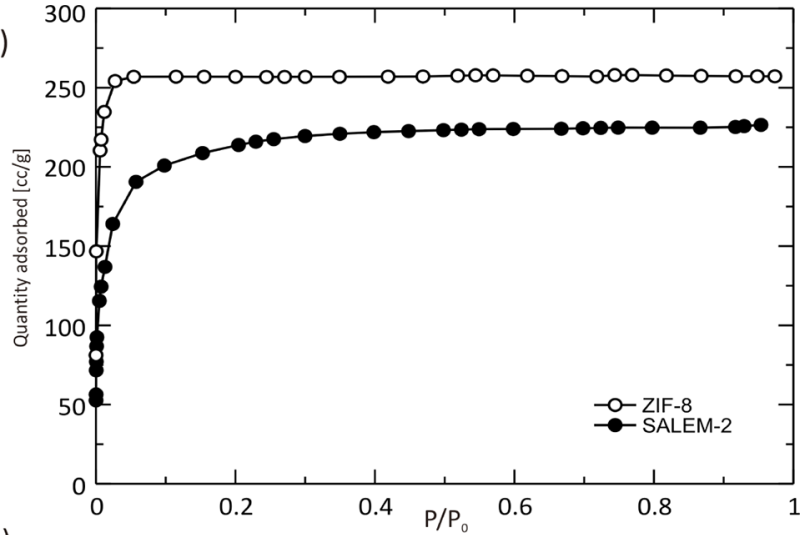

d)

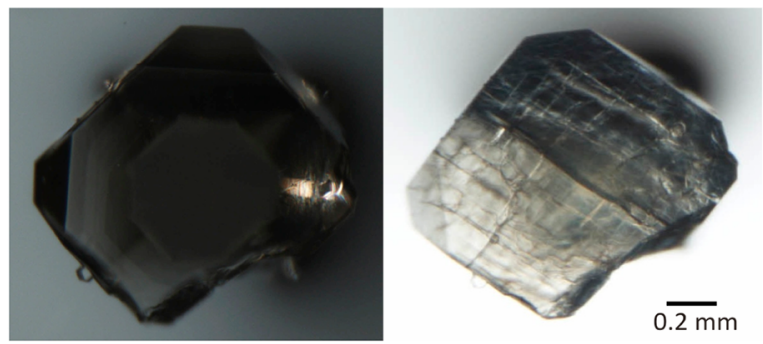

Figure 1. (a) PXRD patterns of SALEM-2 and ZIF-8. (b) NMR spectrum of SALEM-2 digested in $\mathrm{D}_{2} \mathrm{SO}_{4}$ after 7 days of immersion in an excess Him solution (with molar ratio of ZIF-8 to im=1:6.7). (c) $\mathrm{N}_{2}$ isotherms of SALEM-2 and ZIF-8 taken at $77 \mathrm{~K}$. (d) A ZIF-8 crystal (left); the same crystal transformed to SALEM-2 after 7 days in excess $i m$ solution (right).

Table 2. Structural Characteristics of ZIF-8 and SALEM-2

\begin{tabular}{|c|c|c|c|c|c|c|}
\hline ZIF & composition & zeolite topology & space group & unit cell side length $[\AA]$ & solvent-accessible volume $^{a}[\%]$ & BET surface area $\left[\mathrm{m}^{2} \mathrm{~g}^{-1}\right]$ \\
\hline ZIF-8 & $\mathrm{Zn}(\mathrm{mim})_{2}$ & SOD & $I-43 m$ & $16.9910(2)$ & 47.6 & 1080 \\
\hline SALEM-2 & $\mathrm{Zn}(\mathrm{im})_{1.7}(\mathrm{mim})_{0.3}$ & SOD & $I-43 m$ & $16.8303(2)$ & 50.8 & 830 \\
\hline
\end{tabular}

${ }^{a}$ Calculated by PLATON.

\section{RESULTS AND DISCUSSION}

Synthesis and Characterization. Because ZIF-8 is a more chemically robust system than CdIF-4, activating it to SALE required rather forcing conditions. First, we found that the use of $N, N$-dimethylformamide and $N, N$-dimethylacetamide as solvents during the SALE experiment with ZIF-8 led to the degradation of the SOD framework as was demonstrated by PXRD data (Figure S4). The less basic $n$-butanol was found to be a more appropriate solvent for the SALE of im into ZIF-8. Second, the necessary molar ratio of $i m$ to ZIF-8 was found to be 6.7:1 (as compared to the much lower linker-to-ZIF molar ratio of $\sim 3: 1$ found to be sufficient when performing SALE on CdIF-4); ratios higher than 7:1 led to the loss of the SOD topology, while lower ratios did not yield observable linker exchange. SALE was performed by letting ZIF-8 react with im solvothermally at $100{ }^{\circ} \mathrm{C}$ for 7 days (as opposed to only 2 days required for the SALE of CdIF-4 to reach 100\%).

Reaction progress was monitored by ${ }^{1} \mathrm{H}$ NMR; after 7 days, the integration of the $\mathrm{im}$ and $\mathrm{mim}$ signals revealed that $85 \%$ of the ligands present in the system were $\mathrm{im}$. Attempts to perform the reaction for longer than 7 days did not lead to an appreciable increase of the percentage of the im linkers in the system. Monitoring the progress of the SALE reaction by NMR reveals that the linker exchange to im takes place gradually, reaching $80 \%$ by the fourth day of the reaction. The rate of exchange markedly slows after the fourth day, eventually plateauing around $85 \%$ by day seven (Figure S3).

The linker-exchanged material was purified by Soxhlet extraction and activated before measuring its BET surface area $\left(830 \mathrm{~m}^{2} / \mathrm{g}\right)$. PXRD of the material confirmed the retention of the SOD topology, and the size and morphology of the crystals did not change in an observable manner during the course of the reaction (Figure 1). The BET surface area of SALEM-2 appears lower than the surface area of ZIF-8 (which has been reported to be between 1000 and $1700 \mathrm{~m}^{2} / \mathrm{g}$, and is thus highly dependent on the preparation and activation methods employed, Table 2). ${ }^{1,22,54}$ Optimization of the activation procedure for SALEM-2 (including but not limited to Soxhlet extraction in various solvents, solvent exchange, evacuation at higher temperatures, and supercritical drying) may lead to an improvement in its BET surface area.

SALEM-2 exhibits very good thermal stability, as TGA data show no mass is lost until the temperature exceeds about 400 ${ }^{\circ}$ C. Moreover, SALEM-2 remains crystalline after $24 \mathrm{~h}$ in boiling water (Figure S6). Reversibility of the SALE reaction appears feasible; up to $80 \%$ of the im linkers of SALEM-2 could be replaced with mim linkers under SALE conditions with retention of the SOD topology (Figures S7 and S8). Given the fact that the $\mathrm{p} K_{\mathrm{a}}$ values of Hmim and Him are similar (7.52 and 6.97, respectively), the zinc-nitrogen bonds in ZIF-8 and 
SALEM-2 should be of similar strength, and the single-crystal to single-crystal transformation can proceed on the basis of the postulation that we previously proposed. ${ }^{46}$

The final confirmation of successful linker exchange was provided by single-crystal X-ray measurements. The crystal structure of SALEM-2 reveals shared space group and zeolite topology with the parent ZIF-8. The im molecules replace the mim linkers and form 6-linker rings with the alternating $\mathrm{C} 2$ / C4,C5 pattern that is characteristic of ZIF-8. ${ }^{1} \mathrm{H}$ NMR data imply that, while exchange is extensive, roughly one linker in seven remains unexchanged. A close examination of the available X-ray data reveals residual ordered electron density near imidazolate $\mathrm{C} 2$ atoms. We find a superior structural fit if we include $15 \% / 85 \%$ mixed occupancy by methyl and $H$, proximal to $\mathrm{C} 2 .{ }^{55}$ On the basis of these results, we favor this interpretation over an otherwise attractive one that assumes $100 \%$ exchange of $i m$ for mim, but with retention (trapping) of significant amounts of $\mathrm{mim}$ within the framework cavities. ${ }^{56}$

Porosity. It is interesting to note that the departure of the mim linkers opens an aperture along the $a$ crystallographic axis in SALEM-2 (through the four-linker ring) that in ZIF-8 is blocked by methyl groups. Whereas ZIF-8 contains only one aperture type (a six-linker ring, which lies in the (111) plane), SALEM-2 has two (3.4 and $2.4 \AA$ diameter, assuming a van der Waals radius of $1.2 \AA$ for each framework hydrogen atom). Given the fact that, at least in native ZIF-8, the apertures are known to possess a high degree of flexibility (increasing up to $5.8 \AA$ to accommodate transport of larger molecules), ${ }^{57}$ the secondary apertures of SALEM-2 ought to provide an additional means of access to the MOF interior by small molecules such as $\mathrm{CO}_{2}, \mathrm{~N}_{2}$, or $\mathrm{H}_{2}$.

Although the single-crystal XRD data unambiguously confirm the retention of the SOD topology in SALEM-2, they do not provide a straightforward answer to the question of whether the primary aperture (6-linker ring) is larger than in ZIF-8. The ambiguity is mainly due to the fact that the renowned flexibility associated with imidazolate-derived linkers in the SOD structure is not reflected in the crystallographic data. To determine whether the SALE linkers indeed open the ZIF and render the interior of SALEM-2 accessible to molecules too large to pass through the apertures of ZIF-8, we resorted to TGA-MS. This powerful technique can both monitor the departure of guest molecules (via mass loss) and confirm their identity (via electron ionization mass spectrometry).

Activated (solvent evacuated) samples of ZIF-8 and SALEM2 were soaked in $n$-hexane, cyclohexane, or toluene for $24 \mathrm{~h}$, and thereupon analyzed by TGA-MS during heating from 25 to $300{ }^{\circ} \mathrm{C}$. As shown by the TGA data in Figure 2, ZIF-8 releases, and therefore is initially permeated by, only $n$-hexane (kinetic diameter $4.3 \AA$ ). Permeation by $n$-hexane but not cyclohexane is consistent with previous studies of ZIF-8 in the context of chemical sensing. ${ }^{14}$ SALEM-2 likewise accommodates $n$-hexane, but also takes up and releases both cyclohexane (kinetic diameter 6.0 ̊) and toluene (kinetic diameter 6.1 ). It is also worth noting that it takes up almost $50 \%$ more $n$ hexane than does ZIF-8. Finally, MS data (Table S2) confirm that the mass losses exhibited by the ZIF samples are due to the departure of the candidate guest molecules to which the activated ZIFs were exposed. That some peaks appear at temperatures that exceed the boiling points of the guest solvents can be attributed to the microporosity of the ZIFs; it is not uncommon to observe that molecules trapped inside
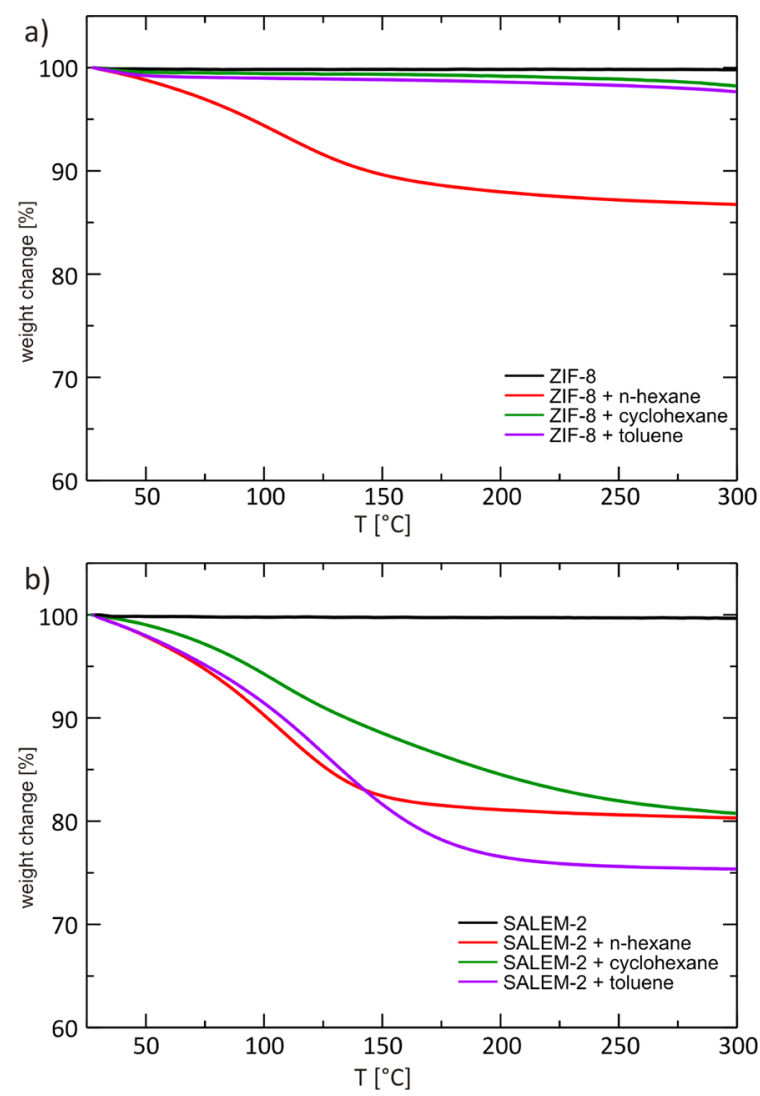

Figure 2. TGA data for ZIF-8 and SALEM-2 soaked in $n$-hexane, cyclohexane, or toluene.

microporous MOFs require additional thermal energy to leave the framework as the kinetic diameter of the guest molecule approaches the size of the aperture. ${ }^{23}$

Catalysis. Recently, we reported on Brønsted-base catalysis of the conjugate addition of alcohol (Scheme 2) by the MOF

\section{Scheme 2. Conjugate Addition of Alcohol}

Catalysis

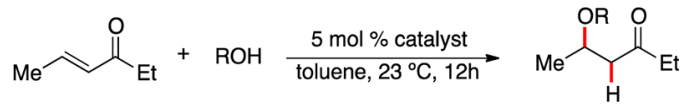

tripodal imidazolate framework-1 (TIF-1), after exposure to $n$ butyllithium and deprotonation (lithiation) of the $\mathrm{C} 2$ site of imidazolates present on the MOF exterior surface. ${ }^{52,58}$ The activated imidazolates constitute $\mathrm{N}$-heterocyclic carbene (NHC)-like entities, but with a transition-metal cation taking the place of one of the nitrogen-bound alkyl groups present on conventional NHC catalyst precursors such as IMes. Remarkably, TIF-1 proved to be a few orders of magnitude more catalytic than IMes on a per-catalyst-site basis, behavior that was attributed primarily to the ability of TIF-1 to preferentially position $\mathrm{Li}^{+}$(a known NHC cocatalyst) proximal to the active site.

We reasoned that upon exposure to $n$-butyllithium, SALEM2 might similarly behave as an NHC-like catalyst, but now with both alkyl groups replaced by transition-metal cations (Scheme 3 ). Thus, from a catalysis perspective, the role of the coordinated zinc ions would be to activate the imidazolate carbons to deprotonation. As summarized in Table 3, 
Scheme 3. Formation of Carbenes and Carbene-like Species in IMes, TIF-1, and SALEM-2
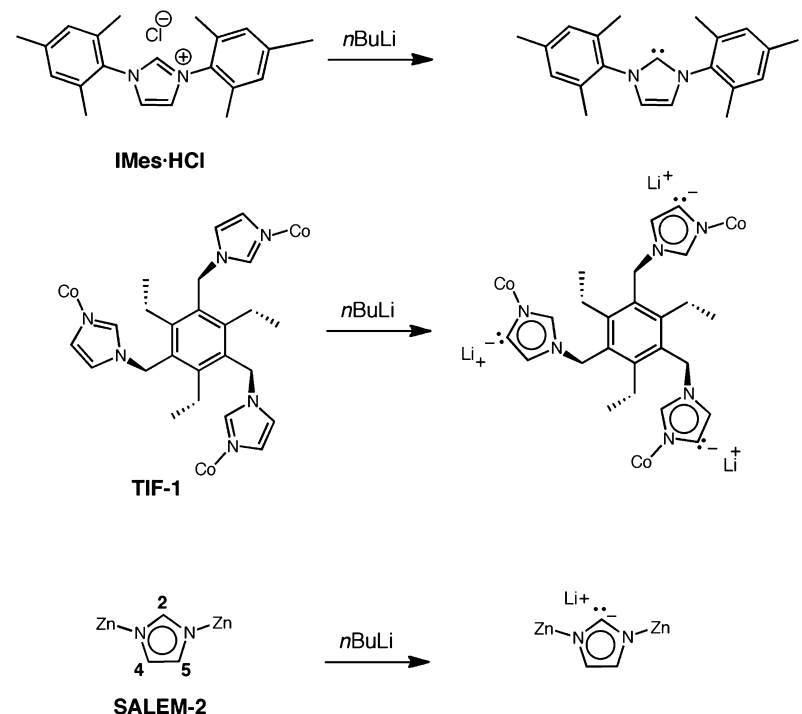

Table 3. Percent Conversion to Corresponding Ethers for SALEM-2, TIF-1, and IMes

\begin{tabular}{lcc}
\multicolumn{1}{c}{ catalyst } & conversion of $\mathrm{BnOH}[\%]$ & conversion of $\mathrm{MeOH}[\%]$ \\
SALEM-2 $^{a}$ & 81 & 94 \\
TIF-1 $^{b}$ & 83 & 65 \\
IMes $^{b}$ & 72 & 77
\end{tabular}

${ }^{a}$ Percent conversion calculated by proton NMR; see Experimental Section for additional details. ${ }^{b}$ Percent conversion calculated by GC.

deprotonated SALEM-2 indeed is catalytic, with the test reactions (as in the TIF-1 study) being the conjugate addition of methanol or benzyl alcohol to the $\alpha, \beta$-unsaturated ketone, 4hex-en-3-one (Scheme 2). As with TIF-1, we assume that the steric demands of both $n$-butyllithium (present in solution as a hexamer) and the products of the conjugate-addition reactions limit catalysis to sites on the external surface of SALEM-2.

To establish that the observed reactivity indeed was catalyzed by SALEM-2 (rather than a molecular moiety), the reaction was repeated, an aliquot was drawn after $1 \mathrm{~h}$, and the remainder of the reaction mixture was filtered through a $100 \mathrm{~nm}$ syringe filter under inert atmosphere. NMR measurements showed no further progression of the reaction with the filtered sample, but continued reactivity with the unfiltered (i.e., ZIF-containing) aliquot. PXRD measurements showed that SALEM-2 remains crystalline following its use as a catalyst (Figure S5).

ZIF-8 showed no catalytic activity when subjected to the same conjugate addition reaction conditions. In contrast to SALEM-2, ZIF-8 is substituted with a methyl group at the C2 position, that is, the site on conventional imidazolium species where a carbene is typically formed. In our work with TIF-1, we were unable to establish with certainty whether the catalytically active site was $\mathrm{C} 2$ or C4 (C5). The ZIF-8/SALEM-2 behavioral difference cements the assignment of $\mathrm{C} 2$ as the active site for catalysis by TIF-1.

\section{CONCLUSIONS}

By using ZIF-8 as a template and solvent-assisted linker exchange (SALE) as an indirect synthesis technique, we have obtained a ZIF of SOD topology that primarily (85\%) contains unsubstituted imidazolate linkers. Despite many attempts, and the prior synthesis of 17 other structurally distinct versions of $\mathrm{Zn}(\mathrm{im})_{2}$, a SOD version had eluded synthesis. The difficulty in preparing a SOD version (i.e., a ZIF-8 structural analogue) by direct synthesis methods is consistent with calculations and observations by Cheetham and co-workers regarding the absolute energies of various polymorphs and correlation of energetic stability with ZIF density. At the same time, the difficulties underscore the potential for SALE to yield desired, but elusive new MOFs.

In contrast to ZIF-8, which features a single aperture type, the new material, SALEM-2, presents apertures defined by both four-linker and six-linker rings. Uptake and release experiments with various candidate guest molecules reveal that SALEM-2 can admit larger guests than can ZIF-8. While consistent with naïve expectations based on replacement of methyl groups by hydrogen atoms, the conclusion that SALEM-2 offers large apertures cannot be drawn from the available single-crystal Xray data alone, as they report only on static structures. For SALEM-2, the crystallographically determined aperture width of $3.4 \AA$ can open sufficiently to admit and release guests having kinetic diameters at least as large as $6.1 \AA$. It will be interesting to see to what extent the more open structure attainable in the SALE-derived material translates into enhanced diffusive transport of molecules such as $n$-alkanes that are capable of permeating ZIF-8.

By offering an accessible imidazolate proton at $\mathrm{C} 2$ of the linkers, SALEM-2 can be reacted with $n$-butyllithium to create a material that is competent for Brønsted base catalysis, as illustrated with simple conjugate-addition reactions. In contrast, ZIF-8, when similarly treated, is not rendered catalytic, a difference that is readily understandable on the basis of the presence of a methyl group at $\mathrm{C} 2$. Because conversion of the SALE-derived ZIF to catalytic form involves $\mathrm{C}-\mathrm{H}$ bond breaking and concomitant creation of a framework-immobilized carbene-like entity, SALEM-2 should be susceptible to further elaboration, for example, via coordination of nonstructural metal ions at the $\mathrm{C} 2$ site. More generally, SALE appears to be a powerful technique for incorporating structurally and chemically attractive linkers into ZIFs, especially in cases where the desired linkers cannot be incorporated de novo. We intend to report shortly on the application of SALE to ZIFs offering other useful topologies.

\section{ASSOCIATED CONTENT}

\section{S Supporting Information}

Single-crystal data for SALEM-2, both assuming 100\% and 85\% exchange to im (CIF, CCDC deposition number 899389); ${ }^{1} \mathrm{H}$ NMR, PXRD, nitrogen sorption, and TGA-MS measurements, chemical stability test results, and reverse SALE experiment data. This material is available free of charge via the Internet at http://pubs.acs.org.

\section{AUTHOR INFORMATION}

\section{Corresponding Author}

j-hupp@northwestern.edu; o-farha@northwestern.edu

Notes

The authors declare no competing financial interest.

\section{ACKNOWLEDGMENTS}

This work made use of the J. B. Cohen X-ray Diffraction Facility supported by the MRSEC program of the National Science Foundation (DMR-1121262) at the Materials Research 
Center of Northwestern University. We gratefully acknowledge DTRA (grant HDTRA1-10-1-0023; catalysis), the Basic Energy Sciences Office of the U.S. Department of Energy (grant DEFG2-08ER15967; selective sorption), and the Polish Ministry of Science and Higher Education through the "Mobility Plus" Program (W.B.) for support of this project.

\section{REFERENCES}

(1) Zhang, J.-P.; Zhu, A.-X.; Lin, R.-B.; Qi, X.-L.; Chen, X.-M. Adv. Mater. 2011, 23, 1268-1271.

(2) Phan, A.; Doonan, C. J.; Uribe-Romo, F. J.; Knobler, C. B.; O'Keeffe, M.; Yaghi, O. M. Acc. Chem. Res. 2009, 43, 58-67.

(3) Zhang, J.-P.; Zhang, Y.-B.; Lin, J.-B.; Chen, X.-M. Chem. Rev. 2011, 112, 1001-1033.

(4) Horike, S.; Shimomura, S.; Kitagawa, S. Nat. Chem. 2009, 1, 695-704.

(5) Farha, O. K.; Hupp, J. T. Acc. Chem. Res. 2010, 43, 1166-1175.

(6) Ferey, G. Chem. Soc. Rev. 2008, 37, 191-214.

(7) Tranchemontagne, D. J.; Mendoza-Cortes, J. L.; O’Keeffe, M.; Yaghi, O. M. Chem. Soc. Rev. 2009, 38, 1257-1283.

(8) Eddaoudi, M.; Moler, D. B.; Li, H.; Chen, B.; Reineke, T. M.; O'Keeffe, M.; Yaghi, O. M. Acc. Chem. Res. 2001, 34, 319-330.

(9) Liu, J.; Thallapally, P. K.; McGrail, B. P.; Brown, D. R.; Liu, J. Chem. Soc. Rev. 2012, 41, 2308-2322.

(10) Shimizu, G. K. H.; Vaidhyanathan, R.; Taylor, J. M. Chem. Soc. Rev. 2009, 38, 1430-1449.

(11) Loeb, S. J. Chem. Soc. Rev. 2007, 36, 226-235.

(12) Banerjee, R.; Phan, A.; Wang, B.; Knobler, C.; Furukawa, H.; O’Keeffe, M.; Yaghi, O. M. Science 2008, 319, 939-943.

(13) Liedana, N.; Galve, A.; Rubio, C.; Tellez, C.; Coronas, J. ACS Appl. Mater. Interfaces 2012, 4, 5016-5021.

(14) Lu, G.; Hupp, J. T. J. Am. Chem. Soc. 2010, 132, 7832-7833.

(15) Li, K.; Olson, D. H.; Seidel, J.; Emge, T. J.; Gong, H.; Zeng, H.;

Li, J. J. Am. Chem. Soc. 2009, 131, 10368-10369.

(16) Chang, N.; Gu, Z.-Y.; Yan, X.-P. J. Am. Chem. Soc. 2010, 132, 13645-13647.

(17) Pan, Y.; Li, T.; Lestari, G.; Lai, Z. J. Membr. Sci. 2012, 390-391, 93-98.

(18) Huang, A.; Bux, H.; Steinbach, F.; Caro, J. Angew. Chem., Int. Ed. 2010, 49, 4958-4961.

(19) Bae, T.-H.; Lee, J. S.; Qiu, W.; Koros, W. J.; Jones, C. W.; Nair, S. Angew. Chem., Int. Ed. 2010, 49, 9863-9866.

(20) Tian, Y.-Q.; Cai, C.-X.; Ji, Y.; You, X.-Z.; Peng, S.-M.; Lee, G.-H. Angew. Chem., Int. Ed. 2002, 41, 1384-1386.

(21) Lobo, R. F.; Zones, S. I.; Davis, M. E.; Herron, N.; Corbin, D. R. J. Inclusion Phenom. Macrocyclic Chem. 1995, 6, 47-78.

(22) Huang, X.-C.; Lin, Y.-Y.; Zhang, J.-P.; Chen, X.-M. Angew. Chem., Int. Ed. 2006, 45, 1557-1559.

(23) Park, K. S.; Ni, Z.; Côté, A. P.; Choi, J. Y.; Huang, R.; UribeRomo, F. J.; Chae, H. K.; O’Keeffe, M.; Yaghi, O. M. Proc. Natl. Acad. Sci. U.S.A. 2006, 103, 10186-10191.

(24) Hayashi, H.; Cote, A. P.; Furukawa, H.; O’Keeffe, M.; Yaghi, O. M. Nat. Mater. 2007, 6, 501-506.

(25) Wang, B.; Cote, A. P.; Furukawa, H.; O’Keeffe, M.; Yaghi, O. M. Nature 2008, 453, 207-211.

(26) Lehnert, R.; Seel, F. Z. Anorg. Allg. Chem. 1980, 464, 187-194.

(27) Tian, Y.-Q.; Zhao, Y.-M.; Chen, Z.-X.; Zhang, G.-N.; Weng, L.H.; Zhao, D.-Y. Chem.-Eur. J. 2007, 13, 4146-4154.

(28) Martins, G. A. V.; Byrne, P. J.; Allan, P.; Teat, S. J.; Slawin, A. M. Z.; Li, Y.; Morris, R. E. Dalton Trans. 2010, 39, 1758-1762.

(29) Lewis, D. W.; Ruiz-Salvador, A. R.; Gomez, A.; RodriguezAlbelo, L. M.; Coudert, F.-X.; Slater, B.; Cheetham, A. K.; MellotDraznieks, C. CrystEngComm 2009, 11, 2272-2276.

(30) Tian, Y.-Q.; Cai, C.-X.; Ji, Y.; You, X.-Z.; Peng, S.-M.; Lee, G.-H. Angew. Chem., Int. Ed. 2002, 41, 1384-1386.

(31) Fairen-Jimenez, D.; Moggach, S. A.; Wharmby, M. T.; Wright, P. A.; Parsons, S.; Düren, T. J. Am. Chem. Soc. 2011, 133, 8900-8902.
(32) Pan, Y.; Liu, Y.; Zeng, G.; Zhao, L.; Lai, Z. Chem. Commun. 2011, 47, 2071-2073.

(33) Cravillon, J.; Münzer, S.; Lohmeier, S.-J.; Feldhoff, A.; Huber, K.; Wiebcke, M. Chem. Mater. 2009, 21, 1410-1412.

(34) Lu, G.; Farha, O. K.; Zhang, W.; Huo, F.; Hupp, J. T. Adv. Mater. 2012, 24, 3970-3974.

(35) Demessence, A.; Boissiere, C.; Grosso, D.; Horcajada, P.; Serre, C.; Ferey, G.; Soler-Illia, G. J. A. A.; Sanchez, C. J. Mater. Chem. 2010 20, 7676-7681.

(36) Wu, Y.-n.; Li, F.; Zhu, W.; Cui, J.; Tao, C.-a.; Lin, C.; Hannam, P. M.; Li, G. Angew. Chem., Int. Ed. 2011, 50, 12518-12522.

(37) Bux, H.; Liang, F.; Li, Y.; Cravillon, J.; Wiebcke, M.; Caro, J. R. J. Am. Chem. Soc. 2009, 131, 16000-16001.

(38) Tan, J. C.; Bennett, T. D.; Cheetham, A. K. Proc. Natl. Acad. Sci. U.S.A. 2010, 107, 9938-9943.

(39) Beldon, P. J.; Fábián, L.; Stein, R. S.; Thirumurugan, A.; Cheetham, A. K.; Friščić, T. Angew. Chem., Int. Ed. 2010, 49, 96409643.

(40) Nguyen, L. T. L.; Le, K. K. A.; Truong, H. X.; Phan, N. T. S. Catal. Sci. Technol. 2012, 2, 521-528.

(41) Kalidindi, S. B.; Esken, D.; Fischer, R. A. Chem.-Eur. J. 2011, 17, 6594-6597.

(42) Chizallet, C.; Lazare, S.; Bazer-Bachi, D.; Bonnier, F.; Lecocq, V.; Soyer, E.; Quoineaud, A.-A.; Bats, N. J. Am. Chem. Soc. 2010, 132, 12365-12377.

(43) Parnham, E. R.; Morris, R. E. Acc. Chem. Res. 2007, 40, 10051013.

(44) Burnett, B. J.; Barron, P. M.; Hu, C.; Choe, W. J. Am. Chem. Soc. 2011, 133, 9984-9987.

(45) Lee, C. Y.; Farha, O. K.; Hong, B. J.; Sarjeant, A. A.; Nguyen, S. T.; Hupp, J. T. J. Am. Chem. Soc. 2011, 133, 15858-15861.

(46) Karagiaridi, O.; Bury, W.; Sarjeant, A. A.; Stern, C. L.; Farha, O. K.; Hupp, J. T. Chem. Sci. 2012, 3, 3256-3260.

(47) Low, J. J.; Benin, A. I.; Jakubczak, P.; Abrahamian, J. F.; Faheem, S. A.; Willis, R. R. J. Am. Chem. Soc. 2009, 131, 15834-15842.

(48) Kim, M.; Cahill, J. F.; Su, Y.; Prather, K. A.; Cohen, S. M. Chem. Sci. 2012, 3, 126-130.

(49) Cavka, J. H.; Jakobsen, S.; Olsbye, U.; Guillou, N.; Lamberti, C.; Bordiga, S.; Lillerud, K. P. J. Am. Chem. Soc. 2008, 130, 13850-13851.

(50) Pangborn, A. B.; Giardello, M. A.; Grubbs, R. H.; Rosen, R. K.; Timmers, F. J. Organometallics 1996, 15, 1518-1520.

(51) Phillips, E. M.; Riedrich, M.; Scheidt, K. A. J. Am. Chem. Soc. 2010, 132, 13179-13181.

(52) Lalonde, M. B.; Farha, O. K.; Scheidt, K. A.; Hupp, J. T. ACS Catal. 2012, 2, 1550-1554.

(53) Because SALEM-2 has 2 imidazoles (2 struts) per asymmetric unit, we loaded SALEM-2 at $2.5 \mathrm{~mol} \%$ as opposed to $5 \mathrm{~mol} \%$ for IMes $\cdot \mathrm{HCl}$ (based on the molecular weight of the asymmetric unit) to maintain the same number of potential catalytic sites per reaction.

(54) Esken, D.; Turner, S.; Wiktor, C.; Kalidindi, S. B.; Van Tenderloo, G.; Fischer, R. A. J. Am. Chem. Soc. 2009, 133, 1637016373.

(55) See the Supporting Information for both versions of CIFs for SALEM-2. The $R$-factors for the $100 \%$ exchanged structure and the structure fitted to $85 \%$ exchange are 2.69 and 2.61, respectively. Residual electron density peaks around the $\mathrm{C} 2$ positions of the im linkers (having roughly $6 \%$ the intensity of the main electron density peaks) suggest the presence of mim in the crystal structure (although it is difficult to assign definitive positions to where this residual linker is found, as the mim linker appears to be randomly interspersed).

(56) The ability of cyclohexane to enter and leave SALEM-2 also argues against persistent trapping of noncoordinated $\mathrm{mim}$ as the explanation for the NMR findings.

(57) Peralta, D.; Chaplais, G.; Simon-Masseron, A.; Barthelet, K.; Chizallet, C.; Quoineaud, A.-A.; Pirngruber, G. D. J. Am. Chem. Soc. 2012, 134, 8115-8126.

(58) Willans, C. E.; French, S.; Anderson, K. M.; Barbour, L. J.; Gertenbach, J.-A.; Lloyd, G. O.; Dyer, R. J.; Junk, P. C.; Steed, J. W. Dalton Trans. 2011, 40, 573-582. 